\title{
Aspectos clínico-patológicos e laboratoriais do envenenamento crotálico experimental em equinos ${ }^{1}$
}

\author{
Cinthia Távora A. Lopes², Carlos Hubinger Tokarnia ${ }^{3}$, Marilene de Farias Brito ${ }^{4}$, \\ Melina Garcia S. de Sousa ${ }^{2}$, Carlos Magno Chaves Oliveira ${ }^{2}$, Natália da Silva e Silva ${ }^{2}$, \\ Danillo Henrique S. Lima ${ }^{2}$ e José Diomedes Barbosa ${ }^{2}$
}

\begin{abstract}
Lopes C.T.A., Tokarnia C.H., Brito M.F. Sousa M.G.S., Oliveira C.M.C., Silva N.S., Lima D.H.S. \& Barbosa J.D. 2012. [Clinical and pathological aspects of experimental crotalic envenoming in horses.] Aspectos clínico-patológicos e laboratoriais do envenenamento crotálico experimental em equinos. Pesquisa Veterinária Brasileira 32(9):843-849. Central de Diagnóstico Veterinário, Universidade Federal do Pará, Rua Maximino Porpino da Silva 1000, Pirapora, Castanhal, PA 68743-080, Brazil. E-mail: cinthiatal@gmail.com

The clinic-pathological picture and laboratory findings in horses experimentally inoculated with the venom of Caudisona durissa terrificus (Crotalus durissus terrificus, according to the former nomenclature) are described. The purpose of this study was to contribute to the understanding of this type of snake accident in horses. The lyophilized venom was diluted into $1 \mathrm{ml}$ of a $0.9 \%$ saline solution and was inoculated subcutaneously into five horses, at the doses of $0.12 \mathrm{mg} / \mathrm{kg}$ (one horse), $0.066 \mathrm{mg} / \mathrm{kg}$ (two horses) and $0.03 \mathrm{mg} / \mathrm{kg}$ (two horses). The venom caused death of the horse that had received $0.12 \mathrm{mg} / \mathrm{kg}$, and of one horse of the two that had received the dose of $0.066 \mathrm{mg} / \mathrm{kg}$. The clinical course varied from $27 \mathrm{~h} 27 \mathrm{~min}$ to $52 \mathrm{~h} 29 \mathrm{~min}$. The second horse inoculated with $0.066 \mathrm{mg} / \mathrm{kg}$ recovered within 12 days after inoculation. The dose of $0.03 \mathrm{mg} / \mathrm{kg}$ had a course of 6 to 10 days, but did not cause fatal envenomation. The clinical picture in the horses was characterized by swelling of the inoculation site (shoulder) that spread to the whole leg, by apathy and lowered head, locomotory alterations shown by dragging of the hoves on the ground, decubitus and difficulty to get up, reduction of auricular, palatal, upperlip and threat reflexes, and increase of heart and breathing frequency. The laboratory examination revealed leukocytosis and lymphocytosis in two horses. There was increase of the creatine-kinase (CK), lactic dehydrogenase (DHL) and urea, and reduction in the seric levels of calcium, phosphorus and magnesium. The activated partial tromboplastina time (TTPA) increased in the horses that died. Postmortem findings were edema of the subcutaneous tissue of the whole leg into which the venom was inoculated, suffusions in the epicard of left and right heart ventricles, and bladder with hemorrhagic areas in its mucosa. Histopatologic examination revealed the liver parenchyma with diffuse moderate vacuolation affecting predominantly the intermediate area of the hepatic lobe, and slight dilation of the sinusoides in some areas, and slight dilation of the kidney tubules mainly in the cortex.
\end{abstract}

INDEX TERMS: Crotalic envenomation, horses, Crotalus sp., Caudisona sp.

\footnotetext{
${ }^{1}$ Recebido em 4 de abril de 2012.

Aceito para publicação em 10 de maio de 2012.

${ }^{2}$ Central de Diagnóstico Veterinário (CEDIVET), Faculdade de Medicina Veterinária, Universidade Federal do Pará (UFPA), Rua Maximino Porpino da Silva 1000, Pirapora, Castanhal, PA 68740-080, Brasil. *Autor para correspondência: cinthiatal@gmail.com

${ }^{3}$ Departamento de Nutrição Animal e Pastagem, Instituto de Zootecnia, Universidade Federal Rural do Rio de Janeiro (UFRRJ), Seropédica, RJ 23890-000, Brasil.

${ }^{4}$ Departamento de Epidemiologia e Saúde Pública, Instituto de Veterinária, UFRRJ, Seropédica, RJ.
}

RESUMO-- Descrevem-se os quadros clínico-patológicos e laboratoriais de equinos inoculados experimentalmente com a peçonha de Caudisona durissa terrificus (Crotalus durissus terrificus na antiga nomenclatura), com a finalidade de fornecer subsídios que favoreçam a compreensão desse tipo de acidente ofídico em equinos. 0 veneno liofilizado foi diluído em $1 \mathrm{ml}$ de solução salina a $0,9 \%$ e inoculado por via subcutânea em cinco equinos, nas doses de $0,12 \mathrm{mg} / \mathrm{kg}$ (um animal), $0,066 \mathrm{mg} / \mathrm{kg}$ (dois animais) e $0,03 \mathrm{mg} / \mathrm{kg}$ (dois animais). 0 veneno causou a morte do equino que recebeu a 
dose de $0,12 \mathrm{mg} / \mathrm{kg}$ e de um dos dois que receberam a dose de $0,066 \mathrm{mg} / \mathrm{kg}$, com evolução de $27 \mathrm{~h} 27 \mathrm{~min}$ e $52 \mathrm{~h} 29 \mathrm{~min}$, respectivamente. 0 segundo animal que recebeu a dose de $0,066 \mathrm{mg} / \mathrm{kg}$ também adoeceu, mas recuperou-se após 12 dias da inoculação. A dose de $0,03 \mathrm{mg} / \mathrm{kg}$ determinou quadros não fatais do envenenamento, com período de evolução que variou entre 6 e 10 dias. 0 quadro clínico caracterizou-se por considerável aumento de volume no local de inoculação (escápula) que se estendeu por todo o membro, apatia e cabeça baixa, alterações locomotoras evidenciadas pelo arrastar das pinças no solo, decúbito e dificuldade para levantar, redução dos reflexos auricular, palatal, do lábio superior e de ameaça, e aumento das frequências cardíaca e respiratória. Os exames laboratoriais revelaram leucocitose por neutrofilia e linfocitose em apenas dois animais. Houve aumento das enzimas creatina quinase (CK), dehidrogenase láctica (DHL) e da ureia, e também redução nos níveis séricos de cálcio, fósforo e magnésio. 0 tempo de tromboplastina parcial ativada (TTPA) aumentou nos equinos que morreram. Os achados de necropsia foram edema do tecido subcutâneo em todo o membro em que foi aplicado o veneno, sufusões no epicárdio dos ventrículos cardíacos esquerdo e direito, e bexiga com áreas hemorrágicas em grande parte da mucosa. Ao exame histopatológico observaram-se fígado com moderada vacuolização difusa, afetando mais a zona intermediária do lóbulo hepático, leve dilatação dos sinusoides hepáticos em algumas áreas e rim com leve dilatação dos túbulos uriníferos, principalmente no córtex.

TERMOS DE INDEXAÇÃO: Envenenamento crotálico, equinos, Crotalus sp., Caudisona sp.

\section{INTRODUÇÃO}

Acidentes com animais peçonhentos são frequentemente relatados nas áreas rurais do Brasil (Silva \& Lofego 2006), onde casos de morte de animais com causa desconhecida são comumente atribuídas a acidentes ofídicos (Tokarnia \& Peixoto 2006).

No Brasil, dentre as 375 espécies de serpentes catalogadas, aproximadamente 60 são peçonhentas e cinco dessas pertencem ao gênero Crotalus, atualmente renomeado para Caudisona (Bérnils \& Costa 2011). Sobre o aspecto de distribuição geográfica, é importante notar que a espécie Caudisona durissa (Crotalus durissus na antiga nomenclatura) habita os cerrados do Brasil central, as regiões áridas e semi-áridas do Nordeste, os campos e áreas abertas do Sul, Sudeste e Norte. A subespécie Caudisona durissa terrificus também se estende pelo oeste, até algumas áreas abertas de Mato Grosso, Rondônia, Amazonas e Pará (campos abertos de Humaitá, Serra do Cachimbo e Santarém). A subespécie Caudisona durissa marajoensis foi descrita nas áreas abertas da Ilha de Marajó, no Pará (Melgarejo 2009).

0 veneno desse gênero de serpentes é composto por uma mistura de proteínas, peptídeos biologicamente ativos, aminas biogênicas e outras substâncias capazes de interferir nos processos fisiológicos (Azevedo-Marques et al. 2009); crotoxina, crotamina, giroxina e convulxina são as principais toxinas isoladas, que produzem três principais efeitos: neu- rotóxico, miotóxico e coagulante; pode ainda haver efeito nefrotóxico e reações edematosas (Silva et al. 1996).

0 diagnóstico de bovinos vitimados por acidente ofídico, causado por serpentes do gênero Caudisona, constitui uma questão de considerável complexidade, uma vez que os sinais clínicos podem ser de difícil interpretação, a evolução ser em geral rápida e à necropsia não haver lesões macroscópicas visíveis (Tokarnia \& Peixoto 2006). Não sabemos qual a situação em relação aos equinos. Desse modo, o objetivo do presente trabalho é descrever os quadros clínico-patológicos e laboratoriais em equinos inoculados experimentalmente pela peçonha de Caudisona durissa terrificus, com a finalidade de fornecer subsídios para o diagnóstico deste envenenamento em equinos.

\section{MATERIAL E MÉTODOS}

Local do experimento e animais. Os experimentos foram realizados entre fevereiro e julho de 2010 em uma propriedade localizada no município de Castanhal, estado do Pará. Foram utilizados sete equinos adultos, sem raça definida, provenientes dos municípios de Capanema e Castanhal, PA, clinicamente sadios, sendo quatro machos e três fêmeas, com pesos variando entre 115 e $327 \mathrm{~kg}$, dentre os quais, dois foram utilizados como controle (Quadro 1). Os animais foram vermifugados e mantidos em pastos de Brachiaria spp. e Panicum spp., com fornecimento de água à vontade.

Procedimento experimental. 0 veneno crotálico utilizado para todos os experimentos proveio de uma só partida obtida no Centro de Estudos de Animais Peçonhentos (CEVAP), Botucatu, SP, o qual foi colhido por extração manual a partir de serpentes da subespécie Caudisona durissa terrificus, com idade, tamanho, peso e procedência variados. Dessa forma, produziu-se um pool que foi dessecado a vácuo e mantido congelado a $17^{\circ} \mathrm{C}$ negativos.

No momento da utilização do veneno, o composto foi reconstituído em solução salina a $0,9 \%$ e inoculado em cinco animais nas doses de $0,12 \mathrm{mg} / \mathrm{kg}$ (um equino), $0,066 \mathrm{mg} / \mathrm{kg}$ (dois equinos) e $0,03 \mathrm{mg} / \mathrm{kg}$ (dois equinos) por via subcutânea, no membro anterior esquerdo, à altura da articulação úmero-rádio-ulnar, com seringa de insulina. Os dois equinos, pertencentes ao grupo controle, receberam $1 \mathrm{ml}$ de solução fisiológica, inoculada pela mesma via e mesmo local de aplicação dos demais. 0 planejamento dos experimentos visou caracterizar os achados clínico-patológicos e laboratoriais do envenenamento crotálico com o menor número possível de animais.

Acompanhamento clínico dos animais. Antes e após a inoculação do veneno realizaram-se exames clínicos dos animais dando-se ênfase ao sistema nervoso. Após a inoculação do veneno, os animais foram examinados a cada duas horas até a morte ou desaparecimento dos sinais clínicos. Executou-se a mesma frequência de exames nos animais controle.

Acompanhamento laboratorial. Para o acompanhamento laboratorial, realizaram-se, em todos os animais, colheitas de sangue nos tempos pré-estabelecidos de $0,2,6,12,24,48$ e 72 horas após a inoculação. Uma vez decorrido esse período, as amostras foram colhidas a cada 24 horas nos animais sobreviventes. Realizou-se, para isso, venopunção da jugular e armazenamento do sangue em frascos diferenciados. Foram utilizados tubos com anticoagulante ácido etilenodiaminotetracético (EDTA) a 10\% para o eritrograma e leucograma; tubos sem anticoagulante adequados à formação, retração do coágulo e obtenção do soro para análises bioquímicas e tubos com anticoagulante protrombina (Doles ${ }^{\circledR}$ ) para a realização do coagulograma. Adicionalmente foi realizada a dosagem de glicose imediatamente após a colheita de sangue por meio de glicosímetro (GlicosímetroAccu-check ${ }^{\circledR}$ Active, Roche). 
Quadro 1. Principais dados dos experimentos em equinos inoculados com veneno crotálico

\begin{tabular}{|c|c|c|c|c|c|c|c|}
\hline $\begin{array}{l}\text { Animal no } \\
\text { (SAP) }\end{array}$ & Peso & Sexo & Dose & $\begin{array}{c}\text { Data e hora } \\
\text { da inoculação }\end{array}$ & $\begin{array}{l}\text { Início dos sinais clínicos } \\
\text { após a inoculação }\end{array}$ & $\begin{array}{l}\text { Tempo de } \\
\text { evolução }\end{array}$ & $\begin{array}{l}\text { Recuperação ou morte } \\
\text { após a inoculação }\end{array}$ \\
\hline Equino 1 & $327 \mathrm{~kg}$ & Fêmea & $0,12 \mathrm{mg} / \mathrm{kg}$ & $\begin{array}{c}25.02 .10 \\
10 \mathrm{~h} 23 \mathrm{~min}\end{array}$ & $14 \mathrm{~min}$ & $27 \mathrm{~h} 27 \mathrm{~min}$ & Morreu \\
\hline Equino 2 & $250 \mathrm{~kg}$ & Macho & $0,066 \mathrm{mg} / \mathrm{kg}$ & $\begin{array}{c}08.07 .10 \\
08 \mathrm{~h} 30 \mathrm{~min}\end{array}$ & $40 \mathrm{~min}$ & $52 \mathrm{~h} 29 \mathrm{~min}$ & Morreu \\
\hline Equino 3 & $200 \mathrm{~kg}$ & Macho & $0,066 \mathrm{mg} / \mathrm{kg}$ & $\begin{array}{c}02.03 .10 \\
13 \mathrm{~h} 51 \mathrm{~min}\end{array}$ & $02 \mathrm{~h} 09 \mathrm{~min}$ & 12 dias & Recuperou-se \\
\hline Equino 4 & $215 \mathrm{~kg}$ & Fêmea & $0,03 \mathrm{mg} / \mathrm{kg}$ & $\begin{array}{l}02.03 .10 \\
13 \mathrm{~h} 55 \mathrm{~min}\end{array}$ & 03h55min & 10 dias & Recuperou-se \\
\hline Equino 5 & $210 \mathrm{~kg}$ & Fêmea & $0,03 \mathrm{mg} / \mathrm{kg}$ & $\begin{array}{c}08.07 .10 \\
08 \mathrm{~h} 41 \mathrm{~min}\end{array}$ & $29 \mathrm{~min}$ & 6 dias & Recuperou-se \\
\hline Controle 1 & $115 \mathrm{~kg}$ & Macho & 1ml solução fisiológica & $\begin{array}{l}02.03 .10 \\
14 \mathrm{~h} 08 \mathrm{~min}\end{array}$ & - & - & Sem sinais clínicos \\
\hline Controle 2 & $177 \mathrm{~kg}$ & Macho & $1 \mathrm{ml}$ solução fisiológica & $\begin{array}{c}02.03 .10 \\
14 \mathrm{~h} 01 \mathrm{~min}\end{array}$ & - & - & Sem sinais clínicos \\
\hline
\end{tabular}

- Sinais clínicos ausentes.

Todas as amostras de sangue foram refrigeradas e transportadas ao Laboratório de Patologia Clínica da Universidade Federal do Pará, onde foram realizados o hemograma e as análises de bioquímica sérica.

O hemograma incluiu a avaliação da série vermelha (hematimetria, hematócrito, hemoglobina, concentração de hemoglobina globular média (CHGM), hemoglobina globular média (HGM), volume globular médio (VGM) e da série branca (leucometria total e leucometria específica). Nos exames de bioquímica sérica foram dosadas ureia, creatinina, cálcio, fósforo, magnésio, AST (aspartato aminotransferase), ALT (alanina aminotransferase), GGT ( $\gamma$-glutamil-transferase), fosfatase alcalina (FA), bilirrubina direta (BD), bilirrubina total (BT), creatina quinase (CK) e deshidrogenase lática (DHL). Para a realização desses exames foram utilizados kits comerciais (Bioclín ${ }^{\circledR}$, Cepa $^{\circledR}$, Doles ${ }^{\circledR}$ ) e as leituras foram realizadas em espectofotômetro (Bioplus, modelo Bio 2000). Na avaliação do coagulograma realizaram-se as seguintes análises: tempo de ativação da protrombina (TAP) e tempo de tromboplastina parcial ativada (TTPA) utilizando-se kits comerciais (Labor$\mathrm{lab}^{\circledR}$ ) com determinação manual.

Necropsias. As necropsias foram realizadas imediatamente após a morte dos animais, com colheita de fragmentos de órgãos, inclusive dos músculos estriados: masseter, língua, cervical, longíssimo dorsal, diafragma, intercostal, bíceps, psoas, semitendinoso, semimembranoso, e pele com tecido subcutâneo e músculos do local da inoculação. As amostras foram fixadas imediatamente em formol a 10\%, com exceção dos fragmentos de músculos, os quais foram previamente expostos ao ambiente por duas horas.

Histopatologia. Os fragmentos foram submetidos ao processamento de rotina para histopatologia, cortados a $5 \mu$ e corados pela hematoxilina e eosina, no Setor de Anatomia Patológica do Convênio Projeto Sanidade Animal Embrapa/Universidade Federal Rural do Rio de Janeiro.

\section{RESULTADOS}

Os principais dados sobre o delineamento experimental encontram-se no Quadro 1.

Dose letal e evolução. A peçonha de Caudisona durissa terrificus causou a morte do equino que recebeu a dose de $0,12 \mathrm{mg} / \mathrm{kg}$ e de um dos dois que receberam a dose de $0,066 \mathrm{mg} / \mathrm{kg}$, com evolução de $27 \mathrm{~h} 27 \mathrm{~min}$ e $52 \mathrm{~h} 29 \mathrm{~min}$, respectivamente. 0 segundo animal que recebeu a dose de 0,066 mg/kg também adoeceu, mas recuperou-se após 12 dias da inoculação. A dose de $0,03 \mathrm{mg} / \mathrm{kg}$ determinou qua- dros não fatais do envenenamento, com período de evolução que variou entre seis e 10 dias.

Quadro clínico geral. Os sinais clínicos iniciaram-se entre $14 \mathrm{~min}$ e $3 \mathrm{~h} 55 \mathrm{~min}$ após a inoculação do veneno. Todos os animais apresentaram considerável aumento de volume no local de inoculação (Fig.1A,B), que se estendia por todo o membro em todos os equinos inoculados, cujo aparecimento variou de $14 \mathrm{~min}$ a $13 \mathrm{~h} 59 \mathrm{~min}$ decorridos da inoculação
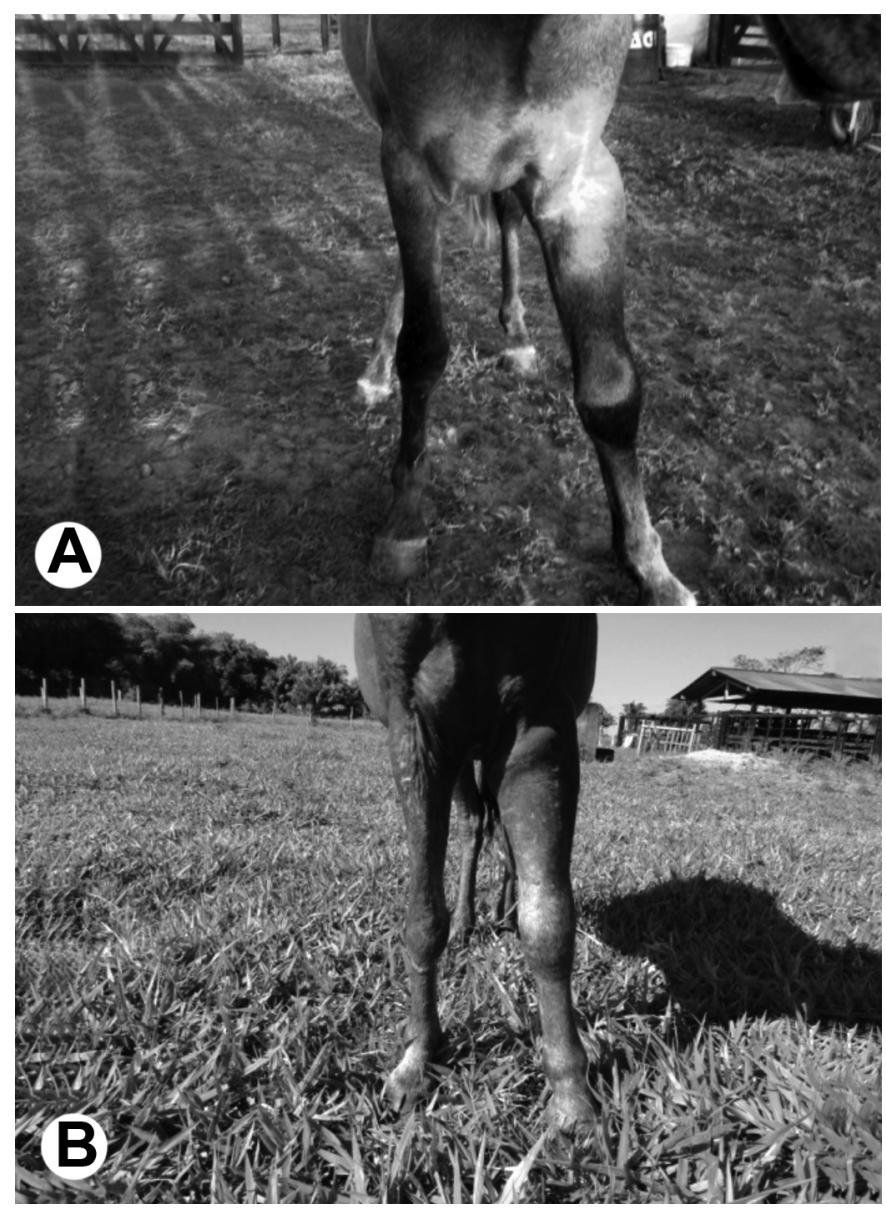

Fig.1A,B. Aumento de volume no membro inoculado no envenenamento crotálico (Equinos 4 e 2, respectivamente). 
do veneno. Esse aumento de volume regrediu em três animais e não mais foi visualizado a partir das $139 \mathrm{~h} 40 \mathrm{~min}(5$ dias), $169 \mathrm{~h} 19 \mathrm{~min}$ (7 dias) e 144h04min (6 dias) (Equinos 3,4 e 5, respectivamente). No membro inoculado, houve, ainda, extravasamento de líquido serossanguinolento pela pele íntegra (Fig.2) da região lateral do metacarpo esquerdo (Equino 2). Foram observados mioclonias (Equino 2), redução da sensibilidade cutânea (Equino 4) e dificuldade de movimentação (Equino 5).

Outros sinais foram apatia (Equinos 1, 3 e 4) (Fig.3), letargia caracterizada por sonolência com tendência em permanecer com a cabeça baixa (Equinos 1, 2 e 5) (Fig.4), edema nos maxilares e acima das pálpebras (Equino 1), ptose auricular (Equino 4), vasos episclerais ingurgitados (Equinos 1, 3, 4 e 5), mucosa ocular hiperêmica (Equinos 3 e 4), sangue nas narinas (Equino 4), dispneia (Equino 1), aumento de linfonodos pré-escapulares e parotídeos (Equinos 4 e 5), submandibulares (Equinos 2, 3 e 5) e pré-crural (Equino 3), e flacidez da cauda (Equino 1).

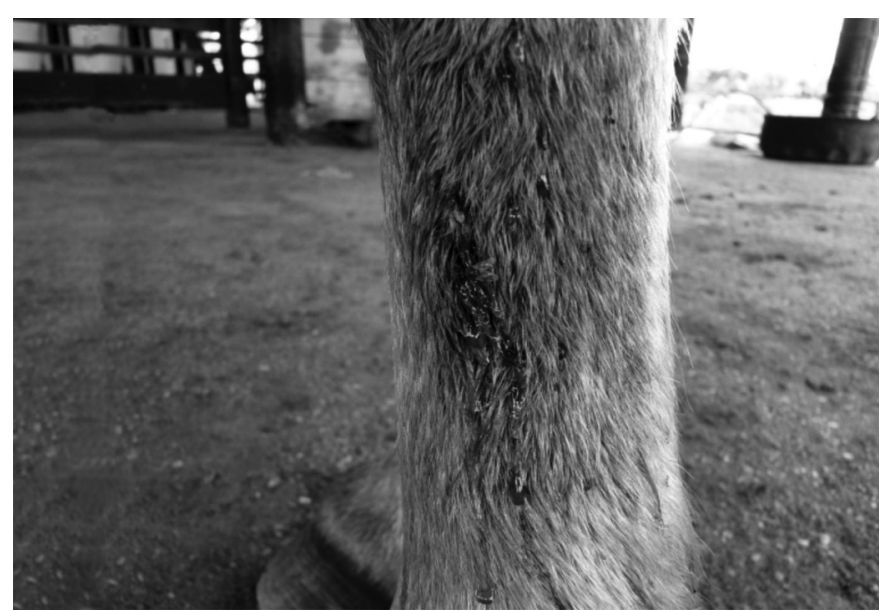

Fig.2. Líquido serossanguinolento drenando da pele íntegra (Equino 2).

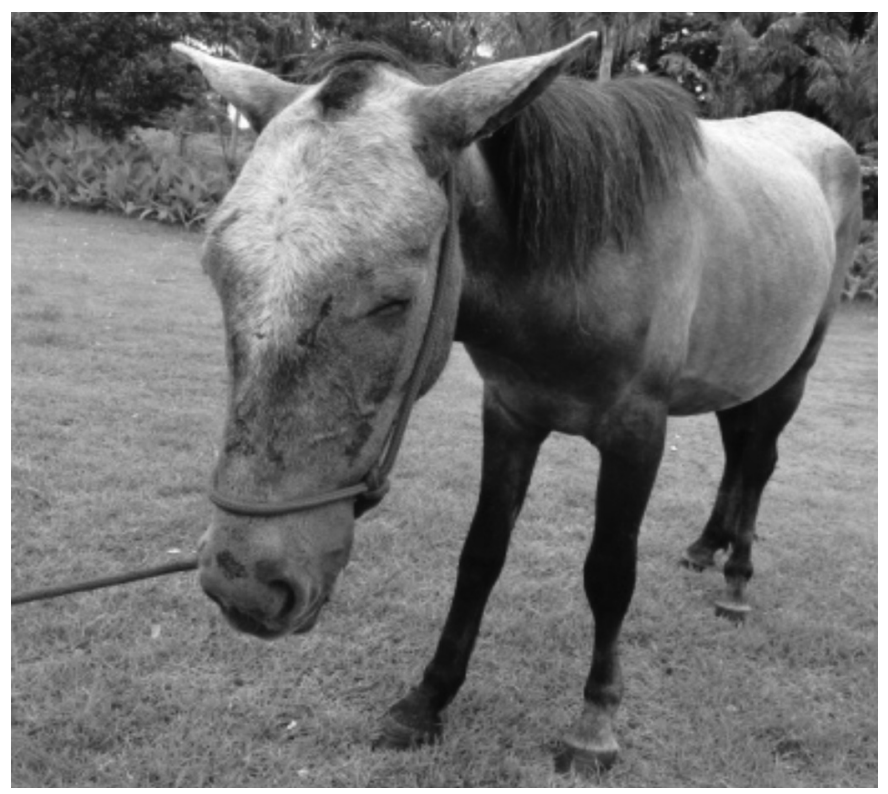

Fig.3. Apatia (Equino 4).

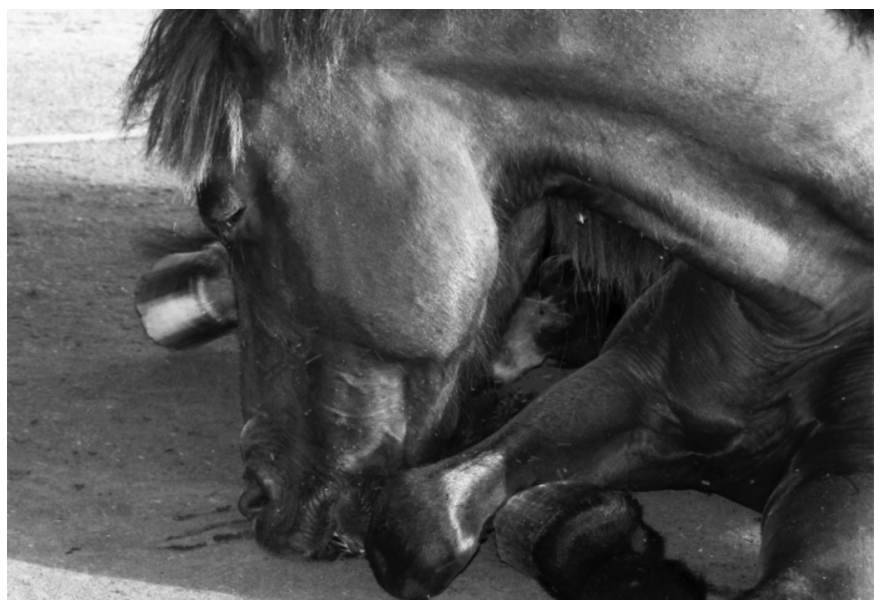

Fig.4. Dificuldade de sustentação da cabeça (Equino 1).

Verificaram-se aumento das frequências cardíaca e respiratória em todos os animais; e temperatura retal diminuída nos Equinos 1, 2 e 4, a partir das $17 \mathrm{~h} 47 \mathrm{~min}, 6 \mathrm{~h}$ e $18 \mathrm{~h}$, respectivamente, após a inoculação do veneno.

Ambos os equinos que morreram (Equinos 1 e 2), urinaram muito no momento da morte.

Outros sinais observados foram arrastar das pinças dos membros anteriores e/ou posteriores durante a caminhada, incoordenação (Equinos 1, 4 e 5), andar cambaleante (Equinos 4 e 5), desequilíbrio (Equino 4), dificuldade de movimentação ao girar em círculo de pequeno raio (Equinos 1, 2 e 5) e demora ou incapacidade de correção da postura durante o teste de abdução e/ou cruzamento de membros (Equinos 3 e 4) (Fig.5). Três dos animais posicionaram-se em decúbito com dificuldade para levantar, mesmo quando estimulados (Equinos 1, 2 e 4).

Os animais, em sua totalidade, apresentaram redução do tônus lingual (Fig.6). Houve, também, redução dos reflexos auricular (Equinos 1, 2 e 4), palatal (Equinos 1, 2, 4 e 5), do lábio superior (Equinos 2, 3 e 5) e de ameaça (Equino 2). Associados a esses sinais observaram-se dificuldade na mastigação e deglutição (Equino 2), acúmulo de alimento na cavidade bucal (Equino 5) e tentativas de execução de movimentos de abertura da boca e estiramento da língua (Equino 3).

Patologia clínica. Na avaliação do eritrograma não foram observadas alterações. No leucograma verificou-se leucocitose em apenas dois animais, caracterizada por neutrofilia e linfocitose (Equinos 3 e 4).

Bioquímica sérica. A elevação dos níveis séricos de ALT foi detectada em dois equinos, a partir das 6h (Equino 4, $16 \mathrm{U} / \mathrm{L}$ ) e das 5h49min (Equino 5, $294 \mathrm{U} / \mathrm{L}$ ). Após um período de $2 \mathrm{~h}$, observou-se aumento da BD nos Equinos 1, 3 e $4(0,76 ; 0,5$ e $1 \mathrm{mg} / \mathrm{dL}$ ) com normalização às $24 \mathrm{~h}$ (Equino 1), $120 \mathrm{~h}$ (5 dias) (Equino 3 ) e às 168h (7 dias) (Equino 4). Verificou-se elevação dos níveis de FA nos Equinos 3, 4 e 5 (cujos maiores valores registrados foram 701, 394 e 463 U/L); elevação da ureia nos Equinos de 1 a 4 (60; 69; 253 e $242 \mathrm{mg} / \mathrm{dL}$ ), com posterior normalização dos valores, exceto no Equino 2; diminuição da creatinina para 0,4 e $0,2 \mathrm{mg} /$ dL (Equinos 2 e 5, respectivamente), com normalização após 120h (5 dias) (apenas do Equino 5) e elevação dessa 


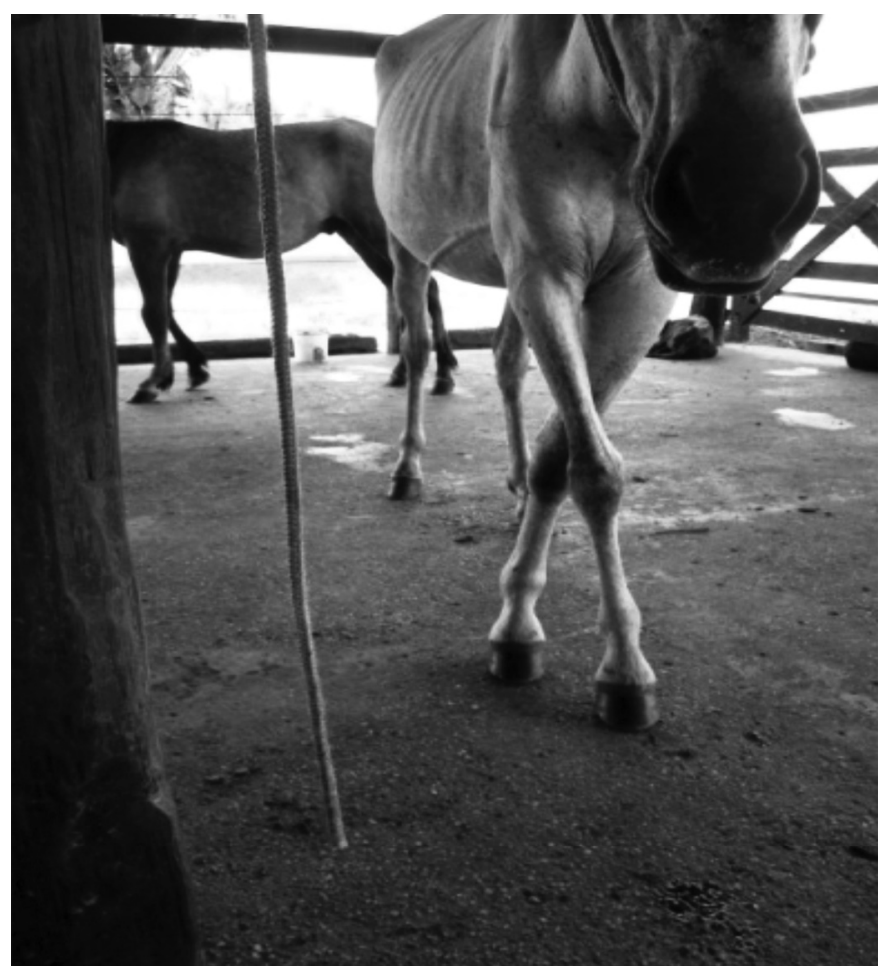

Fig.5. Dificuldade na correção da postura no teste de cruzamento dos membros anteriores (Equino 3).

mesma enzima no Equino 3 (2,4mg/dL), que normalizou-se às $72 \mathrm{~h}$. Houve também elevação das enzimas CK (331; 450; 253; 242 e 396 U/L) e DHL (593; 671; 753; 793 e 712 U/L) em todos os animais, com normalização dos valores de CK apenas nos Equinos 3 e 4 a partir das $120 \mathrm{~h}$ e dos valores de DHL nos Equinos 1, 2, 3 e 5. Observaram-se diminuição dos níveis séricos de cálcio em todos os equinos; diminuição de magnésio nos Equinos 1, 2 e 5 e de fósforo nos Equinos 3 e 4, com posterior normalização.

Observou-se diminuição dos níveis de glicose nos Equinos 1, 2, 3 e 5 (cujos menores valores registrados em cada animal foram $60,44,61$ e $52 \mathrm{mg} / \mathrm{dL}$ ), com elevação dos valores para além do valor de referência nos Equinos 1 e 2 (163 e $227 \mathrm{mg} / \mathrm{dL}$ ) às $24 \mathrm{~h}$ e $48 \mathrm{~h}$, respectivamente, e normalização dos valores no Equino 3.

O tempo de ativação da protrombina (TAP), depois de aumentar por um breve período, diminuiu progressivamente em dois dos animais (Equinos 3 e 4); houve discreto aumento nos Equinos 2 e 5 a partir das $24 \mathrm{~h}$ da inoculação. 0 tempo de tromboplastina parcial ativada (TTPA) diminuiu progressivamente à medida que os animais se recuperavam e aumentou nos Equinos 1 e 2 a partir das $6 \mathrm{~h}$ da inoculação.

Achados de necropsia. Os achados de necropsia foram edema no local da inoculação, estendendo-se às áreas adjacentes a esse ponto (Equino 2) ou por toda a extensão do membro (Equino 1). No coração observaram-se sufusões no epicárdio do ventrículo esquerdo e direito acompanhando o sulco coronário longitudinal (Equino 1) e petéquias no sulco coronário (Equino 2). Verificou-se, ainda, grande área hemorrágica com $20 \mathrm{~cm}$ de diâmetro no útero (Equino 1), bexiga com áreas hemorrágicas em grande parte da mucosa (Equino 2).

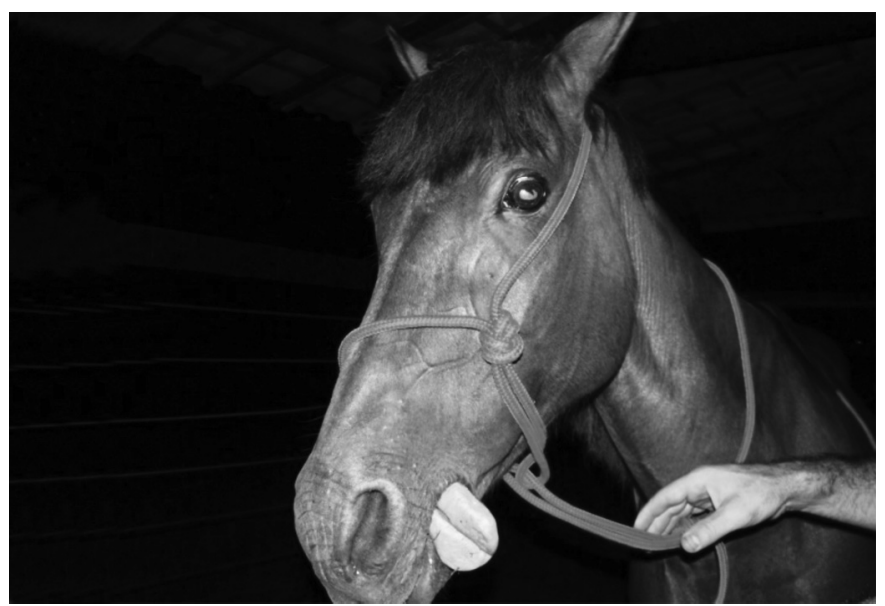

Fig.6. Redução do tônus lingual (Equino 1).

Achados histopatológicos. Ao exame histopatológico observaram-se no fígado moderada vacuolização difusa, que afetava mais intensamente a zona intermediária do lóbulo hepático (Equino 1), e no rim leve dilatação dos túbulos uriníferos, principalmente no córtex (Equino 2).

\section{DISCUSSÃO}

Aspectos toxicológicos e metodologia. Lago et al. (2000) realizaram experimento com a dose de $0,03 \mathrm{mg} / \mathrm{kg}$ do veneno crotálico do tipo crotamina em cinco bovinos fêmeas, a fim de descrever o quadro clínico do envenenamento crotálico nessa espécie. Utilizaram a via intramuscular para inoculação e observaram que, decorridas 20 horas a partir desse procedimento, os animais apresentaram queda progressiva da temperatura retal e morreram. Posteriormente, Graça et al. (2008) reproduziram experimentalmente o envenenamento crotálico através da inoculação do veneno de Caudisona durissa terrificus (cascavel sul-americana) por via subcutânea em 10 bovinos mestiços. Nesse estudo, morreu um bovino inoculado com a dose de $0,03 \mathrm{mg} / \mathrm{kg}$ e quatro de sete bovinos inoculados com a dose de $0,015 \mathrm{mg} / \mathrm{kg}$, enquanto que a dose de $0,0075 \mathrm{mg} /$ $\mathrm{kg}$, aplicada em dois bovinos, provocou apenas sinais discretos com posterior recuperação. Recentemente, Barbosa et al. (2011) em experimento realizado para verificação da sensibilidade de bubalinos e bovinos à peçonha crotálica, inocularam o veneno de Caudisona durissa terrificus em três bubalinos e em dois bovinos utilizando as doses de $0,015,0,03$ e $0,066 \mathrm{mg} / \mathrm{kg}$. 0 bubalino inoculado com a dose de $0,015 \mathrm{mg} / \mathrm{kg}$ recuperou-se em 48 horas; o que recebeu a dose de $0,03 \mathrm{mg} / \mathrm{kg}$ apresentou sinais clínicos graves e recuperou-se em 6 dias, e a dose de $0,066 \mathrm{mg} / \mathrm{kg}$ causou a morte de um terceiro bubalino. Os dois bovinos inoculados com 0,03 e $0,066 \mathrm{mg} / \mathrm{kg}$ do veneno morreram. Desse modo, baseado nos trabalhos acima citados, optou-se em iniciar os experimentos em equinos com a inoculação da dose de $0,03 \mathrm{mg} / \mathrm{kg}$ descrita como letal para bovinos.

Quadro clínico geral. Nos experimentos de Graça et al. (2008), dos sete bovinos que receberam a dose de $0,015 \mathrm{mg} /$ $\mathrm{kg}$, quatro morreram. 0 dobro dessa dose $(0,03 \mathrm{mg} / \mathrm{kg})$, quando inoculada nos equinos deste experimento, produ- 
ziu um quadro clínico mas não foi suficiente para provocar a morte dos equinos deste experimento. Desse modo, enquanto a dose de $0,015 \mathrm{mg} / \mathrm{kg}$ do veneno crotálico pode ser letal para bovinos, para os equinos desse experimento, a mínima dose capaz de causar a morte de animais foi de $0,066 \mathrm{mg} / \mathrm{kg}$ (causou a morte de um de dois equinos).

Dentre os sinais clínicos descritos por Graça et al. (2008) com a peçonha das cascavéis sul-americanas em bovinos, alguns se assemelham aos observados nos cinco equinos inoculados experimentalmente no presente trabalho. Esses sinais incluem dificuldade de locomoção, andar cambaleante e incoordenação motora, além de decúbito esternal e abdominal lateral, apatia, mioclonias, imobilidade do globo ocular e dispneia.

A drenagem do líquido serossanguinolento através da pele íntegra observada no Equino 2 pode estar associada ao nível elevado de prostaglandinas decorrente das ações do veneno e que levam ao aumento da permeabilidade capilar e perda de líquido para os tecidos (Lago et al. 2001).

As alterações neurológicas possivelmente foram provocadas pela ação neurotóxica do veneno, atribuída fundamentalmente à crotoxina que é responsável pelo elevado efeito tóxico do veneno crotálico. A crotoxina constitui uma neurotoxina pré-sináptica que atua nas terminações nervosas motoras, inibindo a liberação de acetilcolina pelos impulsos nervosos. Dessa maneira provoca o bloqueio neuromuscular e, portanto, é responsável pelas paralisias motoras e respiratórias observadas nos animais (Azevedo-Marqueset al. 2009). Nesse contexto, Pinho \& Pereira (2001) citam a ptose palpebral, flacidez da musculatura facial e paralisia velopalatina, também observadas nos equinos desse trabalho.

Resultados laboratoriais. As alterações nos leucogramas dos equinos corroboram com a leucocitose por neutrofilia e por linfocitose citadas por Lago et al. (2001) em bovinos submetidos ao envenenamento crotálico.

Conforme Azevedo-Marques et al. (2009), a ação coagulante do veneno crotálico é atribuída à presença de componente enzimático tipo trombina, cuja propriedade de prolongar o tempo de coagulação ou de tornar o sangue incoagulável se assemelha à encontrada no gênero Bothrops (Azevedo-Marques et al. 2009). Nesse contexto, os testes de coagulação sanguínea demonstraram, nos equinos que morreram, aumento no tempo de ativação da protrombina (apenas Equino 2) e aumento no tempo de tromboplastina parcial ativada (Equinos 1 e 2).

Em relação à ação miotóxica do veneno, atualmente atribuída à crotoxina e à crotamina, elevações intensas da $\mathrm{CK}$, aldolase e AST foram constatadas em pacientes humanos, vítimas de acidente crotálico na década de 80 (Azevedo-Marques et al. 2009). Sobre essas enzimas, verificou-se, igualmente, a elevação da CK em todos os equinos e da DHL nos Equinos 1 a 4, o que pode ser sugestivo de miotoxicidade. No entanto, não foram verificadas elevações da AST e os achados histopatológicos não apontaram lesões microscópicas da musculatura esquelética.

Achados de necropsia e histopatológicos. Graça et al. (2008) verificaram edema quase imperceptível no local da inoculação em bovinos, porém, ao contrário do observado por esses autores, nos equinos do presente estudo observou-se edema considerável, que não ficou restrito ao local da inoculação, mas se estendeu desde a articulação úmero-rádio-ulnar até a extremidade proximal do metacarpo do membro inoculado.

Castro (2006) cita que o acometimento renal é precoce em envenenamento crotálico, sendo o rim o primeiro órgão em que o veneno é detectado. Além disso, a insuficiência renal aguda (IRA) decorrente desse acidente ofídico pode se manifestar clinicamente por oligúria ou anúria e microscopicamente por glomerulonefrite aguda, necrose tubular aguda e necrose cortical renal, entretanto, sem alterações histológicas em alguns casos. Sobre esse aspecto, um único possível indicativo microscópico da ocorrência de lesão renal foi a dilatação dos tubos uriníferos do Equino 2. Entretanto, deve-se considerar que os testes de bioquímica sérica desse animal não apontaram significância na relação ureia-creatinina.

\section{CONCLUSÕES}

As doses tóxicas necessárias para causar a morte dos equinos foram superiores àquelas capazes de causar a morte dos bovinos e bubalinos.

Entretanto, o quadro clínico patológico observado nos equinos foi semelhante ao apresentado nos ruminantes, porém o edema no local da inoculação do veneno, que nos bovinos foi discreto, nos equinos foi considerável.

Agradecimentos.- Ao Centro de Estudos de Animais Peçonhentos-CEVAP, Botucatu, SP e ao Conselho Nacional de Desenvolvimento Científico e Tecnológico-CNPq.

\section{REFERÊNCIAS}

Azevedo-Marques M.M., Hering S.E. \& Cupo P. 2009. Acidente crotálico, p.91-98. In: Cardoso J.L.C., França F.O.S., Wen F.H., Málaque C.M.S. \& Haddad Jr V. (Eds), Animais Peçonhentos no Brasil: Biologia, Clínica e Terapêutica dos Acidentes. Sarvier Editora, São Paulo.

Barbosa J.D., Sousa M.G.S., Tokarnia C.H., Brito M.F., Belo-Reis A.S., Bomjardim H.A., Lopes C.T.A. \& Oliveira C.M.C. 2011. Quadro clínico-patológico do envenenamento crotálico experimental em bubalinos comparado com o de bovinos. Pesq. Vet. Bras. 31(11):967-973.

Bérnils R.S \& Costa H.C. Brazilian reptiles: List of species. Sociedade Brasileira de Herpetologia. Disponível em <http://sbherpetologia.org.br> Acesso 27 set. 2011.

Castro I. 2006. Estudo da toxicidade das peçonhas crotálicas e botrópicas, no acidente ofídico, com ênfase na toxicidade renal. 0 Mundo da Saúde 30(4):644-653.

Graça F.A.S., Peixoto P.V., Coelho C.D., Caldas S.A. \& Tokarnia C.H. 2008. Aspectos clínico-patológicos e laboratoriais do envenenamento crotálico experimental em bovinos. Pesq. Vet. Bras. 28(6):261-270.

Lago L.A., Ferreira P.M., Facury Filho E.J., Melo M.M. \& Alzamora Filho F. 2000. Quadro clínico do envenenamento crotálico experimental em bovinos (Crotalus durissus terrificus - crotamina positivo). Braz. J. Vet. Res. Anim. Sci. 37(4):312-315.

Lago L.A., Melo M.M., Ferreira P.M. \& Facury Filho, E.J. 2001. Alterações hematológicas em bovinos submetidos ao envenenamento crotálico. Revta Bras. Saúde Prod. Anim. 1(1):7-13.

Melgarejo A.R. 2009. Serpentes peçonhentas do Brasil, p.42-69. In: Cardoso J.L.C., França F.O.S., Wen F.H., Málaque C.M.S. \& Haddad Jr V. (Eds), Animais Peçonhentos no Brasil: biologia, clínica e terapêutica dos acidentes. Sarvier Editora, São Paulo. 
Pinho F.M. \& Pereira I.D. 2001. Ofidismo. Assoc. Med. Brasil 47(1):24-29.

Silva M.D., Resende L.A.L., Ueda K.A., Barraviera B., Mendes R.P. \& Montenegro M.R.G. 1996. Ação neuro-muscular do veneno crotálico. Arq. Neuropsiquiatr. 54(1):1-11.
Silva E.R.O. \& Lofego A.C. 2006. Acidentes ofídicos na região de São José do Rio Preto, SP. Revta Unorp 13:127-133.

Tokarnia C.H. \& Peixoto P.V. 2006. A importância dos acidentes ofídicos como causa de mortes em bovinos no Brasil. Pesq. Vet. Bras. 26(2):55-68. 